\title{
Excessive Extracellular Volume Reveals a Neurodegenerative Pattern in Schizophrenia Onset
}

\author{
Ofer Pasternak, ${ }^{1}$ Carl-Fredrik Westin, ${ }^{2}$ Sylvain Bouix, ${ }^{1}$ Larry J. Seidman, ${ }^{4,6}$ Jill M. Goldstein, ${ }^{1,3,4}$ Tsung-Ung W. Woo, ${ }^{5}$ \\ Tracey L. Petryshen, ${ }^{6,7,8}$ Raquelle I. Mesholam-Gately, ${ }^{4}$ Robert W. McCarley, ${ }^{4,9}$ Ron Kikinis, ${ }^{2}$ Martha E. Shenton, ${ }^{1,9}$ and \\ Marek Kubicki ${ }^{1}$ \\ Departments of ${ }^{1}$ Psychiatry, ${ }^{2}$ Radiology, and ${ }^{3}$ Medicine, Brigham and Women's Hospital and Harvard Medical School, Boston, Massachusetts 02115, \\ ${ }^{4}$ Massachusetts Mental Health Center, Public Psychiatry Division, Beth Israel Deaconess Medical Center, Harvard Medical School, Boston, Massachusetts \\ 02215, ${ }^{5}$ Department of Psychiatry, McLean Hospital, Harvard Medical School, Belmont, Massachusetts 02478, ${ }^{6}$ Department of Psychiatry, Massachusetts \\ General Hospital, Harvard Medical School, Boston, Massachusetts 02114, ${ }^{7}$ Stanley Center for Psychiatric Research, Broad Institute of MIT and Harvard, \\ Boston, Massachusetts 02142, ${ }^{8}$ Psychiatric and Neurodevelopmental Genetics Unit, Center for Human Genetic Research, Massachusetts General Hospital, \\ Boston, Massachusetts 02144, and ${ }^{\circ}$ Department of Psychiatry, Veterans Affairs Boston Healthcare System, Brockton Division, Brockton, Massachusetts
} 02301

Diffusion MRI has been successful in identifying the existence of white matter abnormalities in schizophrenia in vivo. However, the role of these abnormalities in the etiology of schizophrenia is not well understood. Accumulating evidence from imaging, histological, genetic, and immunochemical studies support the involvement of axonal degeneration and neuroinflammation-ubiquitous components of neurodegenerative disorders - as the underlying pathologies of these abnormalities. Nevertheless, the current imaging modalities cannot distinguish neuroinflammation from axonal degeneration, and therefore provide little specificity with respect to the pathophysiology progression and whether it is related to a neurodegenerative process. Free-water imaging is a new methodology that is sensitive to water molecules diffusing in the extracellular space. Excessive extracellular volume is a surrogate biomarker for neuroinflammation and can be separated out to reveal abnormalities such as axonal degeneration that affect diffusion characteristics in the tissue. We applied free-water imaging on diffusion MRI data acquired from schizophrenia-diagnosed human subjects with a first psychotic episode. We found a significant increase in the extracellular volume in both white and gray matter. In contrast, significant signs of axonal degeneration were limited to focal areas in the frontal lobe white matter. Our findings demonstrate that neuroinflammation is more prominent than axonal degeneration in the early stage of schizophrenia, revealing a pattern shared by many neurodegenerative disorders, in which prolonged inflammation leads to axonal degeneration. These findings promote anti-inflammatory treatment for early diagnosed schizophrenia patients.

\section{Introduction}

The diffusion MRI methodology of diffusion tensor imaging (DTI) and its unique ability to detect microstructural changes in vivo (Basser and Pierpaoli, 1996) provided considerable evidence for white matter abnormalities in patients diagnosed with schizophrenia (Kubicki et al., 2007). Nevertheless, inferring the under-

Received June 18, 2012; revised Aug. 29, 2012; accepted 0ct. 5, 2012.

Author contributions: 0.P., C.-F.W., L.J.S., J.M.G., T.-U.W.W., T.L.P., R.I.M.-G., R.W.M., R.K., M.E.S., and M.K. designed research; 0.P., S.B., T.L.P., R.I.M.-G., M.E.S., and M.K. performed research; O.P., C.-F.W., and S.B. contributed unpublished reagents/analytic tools; 0.P. and S.B. analyzed data; 0.P., M.E.S., and M.K. wrote the paper.

This work was supported by National Institutes of Health Grants P50-MH080272, R01-MH074794, R01MH050740, R01-MH082918, P41-RR013218, and M01-RR01032; a Veterans Affairs (VA) merit award, a VA Center grant, and a National Alliance for Research on Schizophrenia and Depression (NARSAD) distinguished investigator grant (M.E.S). O.P. was partly supported by a Fulbright Fellowship (Fulbright Commission for Israel, the United States-Israel Educational Foundation) and by a NARSAD Young Investigator Grant from the Brain and Behavior Research Foundation.

The authors declare no competing financial interests.

Correspondence should be addressed to Ofer Pasternak, Psychiatry Neuroimaging Laboratory, 1249 Boylston Street, Boston MA 02215. E-mail: ofer@bwh.harvard.edu.

DOI:10.1523/JNEUROSCI.2904-12.2012

Copyright $\odot 2012$ the authors $\quad 0270-6474 / 12 / 3217365-08 \$ 15.00 / 0$ lying pathophysiology of the white matter abnormalities, or their relation to the etiology of schizophrenia, is challenging, since DTI indices are sensitive to a large number of possible pathologies (Assaf and Pasternak, 2008). Two probable pathologies emerge when considering DTI results in schizophrenia in the context of histological, genetic, immunochemical, and other imaging findings: (1) axonal degeneration in the form of alteration to the myelin sheath (Davis et al., 2003; Kubicki et al., 2005; Uranova et al., 2007) and (2) neuroinflammation (Muller and Schwarz, 2006; Potvin et al., 2008; van Berckel et al., 2008; Doorduin et al., 2009; Laan et al., 2009). These two pathologies are a hallmark of neurodegenerative processes (Weiner and Selkoe, 2002), yet distinguishing between the two is required for the development of targeted drugs and to understand the etiology and progression of schizophrenia.

Myelin alterations affect the diffusion anisotropy of water molecules in the vicinity of axons (Song et al., 2003). In contrast, neuroinflammation is expected to affect the interstitial extraneuronal space, where microglia and other immunoreactive cells mediate neuroinflammation (Schwartz et al., 2006), thereby 
increasing isotropically diffusing extracellular water content (Sykova and Nicholson, 2008). However, despite these microstructural differences, the main DTI indices, such as fractional anisotropy (FA) and mean diffusivity (MD), respond similarly to the two pathologies with FA decrease and MD increase (Alexander et al., 2007), a common report in many DTI studies of schizophrenia patients (Kubicki et al., 2007).

To discriminate between axonal degeneration and neuroinflammation in schizophrenia, we applied the previously introduced free-water imaging (Pasternak et al., 2009). The method explicitly estimates the contribution of freely diffusing water molecules (Pierpaoli and Jones, 2004; Pasternak et al., 2009), which is expected to increase with neuroinflammation (Wang et al., 2011). The free-water contribution can be eliminated, and the remaining signal provides a measure of diffusion fractional anisotropy within tissue $\left(\mathrm{FA}_{\mathrm{T}}\right)$ (Pasternak et al., 2009), which is more specific to tissue alterations than FA (Metzler-Baddeley et al., 2011, 2012). We compared a sample of patients within their first psychotic episode with matched healthy controls to identify the early pathology of schizophrenia.

Among DTI studies of first-episode patients, many report widespread white matter abnormalities (Kyriakopoulos and Frangou, 2009; Peters et al., 2010), in contrast with the typically more focal findings in other DTI studies (Kubicki et al., 2007) and, importantly, in histological studies (Uranova et al., 2007) of chronic patients. If schizophrenia is a neurodegenerative disorder, the degeneration is expected to show a progressive and deteriorating pattern (Lieberman, 1999; Andreasen, 2010), in contrast with the current DTI findings. Using free-water imaging, we tested whether distinguishing the effect of neuroinflammation from that of axonal degeneration could explain the extent of findings in patients at the onset of schizophrenia.

\section{Materials and Methods}

Subjects. The subjects were 18 patients within their first psychotic episode and 20 matched healthy controls. The first-episode patients were assessed during their first hospitalization for psychosis or immediately following discharge. Included patients had continuous psychotic symptoms or antipsychotic drug treatment for no longer than 1 year and had a Diagnostic and Statistical Manual of Mental Disorders, fourth edition (DSM-IV), diagnosis of schizophrenia, schizoaffective disorder, or schizophreniform disorder, based on the Structural Clinical Interview for the DSM-IV text revision (DSM-IV-TR). The patients were either not medicated (three patients) or medicated with typical/atypical antipsychotics (Table 1 ). Thirteen of the patients were also medicated on one or more psychiatric medications other than antipsychotics that included anticholinergic drugs (four patients), mood stabilizers (three patients), antidepressants (six patients), antianxiety medications (three patients), and $\beta$-blockers (one patient). Healthy controls were drawn from the same geographic base as the first-episode group with comparable age, gender, education, race, and ethnicity (Table 1). No controls met criteria for any current major DSM-IV-TR Axis I disorders or any history of psychosis, major depression (recurrent), bipolar disorder, obsessive compulsive disorder, post-traumatic stress disorder, or developmental disorders. Controls were also excluded for any history of psychiatric hospitalizations, prodromal symptoms, schizotypal or other Cluster A personality disorders, first-degree relatives with psychosis, or any current or past use of antipsychotics. All subjects met additional exclusion criteria designed to omit neurological comorbidities and substance abuse. All subjects were recruited as part of the Boston Center for Intervention Development and Applied Research project. The local institutional review board committee approved all protocols, and all subjects gave informed written consent before participation.

Imaging parameters. MRI scanning was performed at the Brigham and Women's Hospital in Boston with a 3 tesla General Electric Signa System (GE Medical Systems). The MR sequences had high spatial resolution
Table 1. Clinical and experimental parameters

\begin{tabular}{llll}
\hline & Controls & First episode & $t$ test \\
\hline Number of subjects & 20 & 18 & \\
Female & 5 & 4 & \\
Male & 15 & 14 & \\
Age (years) & $24.05 \pm 3.99$ & $21.61 \pm 4.34$ & $t_{(36)}=-1.88 ; p=0.07$ \\
Education (years) & $13.78 \pm 2.51$ & $14.17 \pm 1.65$ & $t_{(36)}=0.55 ; p=0.59$ \\
Motion (mm) & $0.70 \pm 0.26$ & $0.68 \pm 0.26$ & $t_{(36)}=-0.22 ; p=0.83$ \\
TE (ms) & $85.84 \pm 1.36$ & $85.73 \pm 1.28$ & $t_{(36)}=-0.26 ; p=0.80$ \\
SNR (raw) & $12.06 \pm 1.47$ & $12.12 \pm 1.58 \quad t_{(36)}=0.12 ; p=0.91$ \\
SNR filtered & $13.05 \pm 1.14$ & $13.22 \pm 1.18$ & $t_{(36)}=0.46 ; p=0.65$ \\
CPZ (mg/d) & & $472.24 \pm 651.33$ & \\
Duration of illness (months) & & $6.19 \pm 4.27$ & \\
Duration of medication & & $4.04 \pm 3.58$ & \\
$\quad$ (months) & & & \\
Antipsychotic medication & & $1 / 12 / 2 / 3$ & \\
$\quad$ (typical/atypical/both/none) & & & \\
\hline
\end{tabular}

The $t$ test column holds the $t$ statistic (with degrees of freedom) and significance value, $p$, for an unpaired twosample $t$ test.

and included 3D T1-weighted [inversion recovery spoiled gradient recalled (SPGR)], 3D T2-weighted (CUBE), and diffusion acquisitions. The IR-SPGR sequence had a repetition time (TR) of $7.8 \mathrm{~ms}$, an echo time (TE) of $3 \mathrm{~ms}$, an inversion time of $600 \mathrm{~ms}$, a $10^{\circ}$ flip angle, a field of view (FOV) of $256 \times 256 \mathrm{~mm}$, a matrix size of $256 \times 256,176$ slices, and $1 \mathrm{~mm}$ slice thickness. The 3D T2-weighted sequence had a TR of $3 \mathrm{~s}$, a TE of $90 \mathrm{~ms}$, a $90^{\circ}$ flip angle, a FOV of $256 \times 256 \mathrm{~mm}$, a matrix size of $256 \times$ 256, 176 slices, and $1 \mathrm{~mm}$ slice thickness. The high-resolution diffusion acquisition was twice refocused and had a TR of $17 \mathrm{~s}$, a $90^{\circ}$ flip angle, a FOV of $240 \times 240 \mathrm{~mm}$, a matrix size of $144 \times 144,85$ slices, $1.7 \mathrm{~mm}$ slice thickness, 51 gradient directions with a b-value of $900 \mathrm{~s} / \mathrm{mm}^{2}$, and eight additional non-diffusion-weighted $\left(b_{0}\right)$ images. We used the propriety GE DTI sequence, which on a 3 tesla GE scanner minimizes TE per each subject. The mean TE value was $86 \mathrm{~ms}$ for both groups (Table 1 ). Nevertheless, since TE directly affects image quality, we included it as a covariate in the statistical analyses.

Construction of DTI and free-water maps. The diffusion data were corrected for motion and artifacts by means of affine registration with a reference volume [FLIRT; FMRIB Software Library (FSL), Oxford, UK] and Rician denoising (Aja-Fernandez et al., 2008). Diffusion gradients were compensated for rotations. A relative-motion parameter was estimated from the transformation matrices (Ling et al., 2012). The images were masked to exclude nonbrain areas by manually annotating a label map that was initialized using Otsu's method as implemented in the software 3DSlicer (www.slicer.org). This additional manual step was required to ensure high-quality cross-subject registration that is not biased by masking errors. To account for possible acquisition or filtering biases, signal-to-noise ratio (SNR) values were calculated for the original and for the filtered $b_{0}$ images using the formula SNR $=\mu / \sigma$, where $\mu$ is the mean, and $\sigma$ is the standard deviation over the repeated $b_{0}$ images. The SNR scores were compared across the two groups using a $t$ test and revealed no significant difference (Table 1).

Maps of FA and MD were calculated for each voxel using 3DSlicer. Free-water and $\mathrm{FA}_{\mathrm{T}}$ maps were calculated by fitting the following model in each voxel (Pasternak et al., 2009): $A_{q}(D, f)=f \exp \left(-b q^{\mathrm{T}} D q\right)+(1-$ $f) \exp \left(-b d_{\text {water }}\right)$, where $A_{q}$ is the modeled attenuated signal (normalized by the $b_{0}$ ) for the applied diffusion gradient $q$, and $b$ is the b-value. The first term reflects the tissue compartment, where $D$ is the diffusion tensor of this compartment, and $f$ is the fractional volume of the compartment. The second term reflects an isotropic free-water compartment, with a fractional volume of $1-f$, and $d_{\text {water }}$ is the diffusion coefficient, set to the diffusivity of water in body temperature $\left(3 \times 10^{-3} \mathrm{~mm}^{2} / \mathrm{s}\right)$. Of note, in a typical diffusion MRI acquisition, the free-water diffusivity dictates an average molecule displacement in the range of a few tens of microns, i.e., the signal originates from large enough water pockets, which in the brain could only appear in the extracellular space. The free-water fractional volume is therefore proportional to the extracellular volume (Wang et al., 2011). On the other hand, the signal of the tissue compartment orig- 
inates from all other water molecules. These molecules are hindered or restricted by cellular structures (e.g., membranes and myelin sheaths), which include all of the intracellular molecules and some extracellular molecules that are in proximity to cellular restrictions. Fitting tensors to the tissue compartment therefore provides a better estimate of processes that occur in the tissue (Metzler-Baddeley et al., 2011), thereby reducing the effect of partial-volume with the extracellular compartment (Metzler-Baddeley et al., 2012).

The model was fitted using a regularized minimization process similar to the algorithm of Pasternak et al. (2009) and updated to use a Euclidean metric for tensor distances (Pasternak et al., 2010, 2012). The regularization incorporates a tensor-based operator (Gur et al., 2009) that is appropriate for both isotropic and anisotropic tensors, applicable for both gray and white matter. The regularization is essential to stabilize the model fit, which is ill posed when using conventional single-shell clinical DTI data acquisition, such as that used here. This regularization approach was shown to produce comparable results to more advanced multishell acquisition protocols in which the regularization can be relaxed (Pasternak et al., 2012).

The estimation of the free-water fractional volume, and the diffusion tensor of the tissue compartment, much like the estimation of DTI tensors, can be biased by various variables such as the image resolution, diffusion protocol parameters, acquisition noise, and the underlying T1 or T2 relaxations (Pasternak et al., 2009). Crossing-fibers may also affect the estimation of the parameters. However, preliminary results show that free-water maps generated when accounting for crossing-fibers are similar to maps generated using the method described here (Baumgartner et al., 2012). Therefore, best practice when comparing the free-water derived parameters between groups is to verify that there are no groupwise differences in the potentially biasing parameters, or to include these as control variables in the statistical analysis. Following the estimation of the model, free-water maps were generated by plotting the voxelwise value of $1-f ; \mathrm{FA}_{\mathrm{T}}$ maps were generated by calculating the $\mathrm{FA}$ of the fitted tensor, $D$, in each voxel. These voxels covered the entire masked brain, including white matter, gray matter, and CSF.

White matter skeleton analysis. The FA maps of all subjects were united to build a white matter skeleton, using the Tract Based Spatial Statistics (TBSS) software (Smith et al., 2006). Individual FA, MD, free-water, and $\mathrm{FA}_{\mathrm{T}}$ maps were projected onto the skeleton. A nonparametric permutations-based test (Nichols and Holmes, 2002) between the firstepisode and control groups was performed on the projected data (Randomize, FSL). Threshold-free cluster enhancement (TFCE) (Smith and Nichols, 2009) was used to avoid defining arbitrary cluster-forming thresholds and smoothing levels. The data were tested against an empirical null distribution generated by 5000 permutations for each contrast, thus providing statistical maps fully corrected for multiple comparisons across space. A corrected value of $p<0.05$ was considered significant. The test was linearly adjusted for motion, age, and TE, which were included as covariates. These three covariates are known to affect diffusion measures and were included to prevent from a systematic bias toward one of the groups, even though there was not a significant difference in the average age, motion, and TE between the groups (Table 1). Statistical maps plotting the corrected $p$ values were visualized using FSL in 2D (TBSS-fill and FSLview). 3DSlicer was used for volume rendering. Voxelwise permutation-based partial correlation tests (Randomize, FSL) adjusted for age, motion, and TE were performed to establish the correlation between, on one hand, the free-water or $\mathrm{FA}_{\mathrm{T}}$ values and, on the other hand, chlorpromazine (CPZ) equivalent daily dosage value, duration of medication, Brief Psychiatric Rating Scale (BPRS; a measure of illness severity) rating (Ventura et al., 2000), or duration of illness. The significance threshold of the correlation was set to 0.05 corrected for multiple comparisons.

Brain parcellation analysis. Each anatomical T1 image was masked by manually annotating a label map created by a brain extraction tool (FSL) to include the brain and exclude the skull. The masked images were then automatically segmented into white and gray matter sections using FreeSurfer (surfer.nmr.mgh.harvard.edu). We compared 70 gray matter sections ( 35 in each hemisphere) and 68 white matter sections (34 in each hemisphere) that were consistently recognized in all the subjects. The sections were mapped onto the diffusion space by registering the masked T1 image with the masked T2 image (rigid registration, FLIRT, FSL), and then registering the T2 image with the masked $b_{0}$ image (nonlinear registration, FNIRT, FSL). The mean $\mathrm{MD}, \mathrm{FA}_{\mathrm{T}}$, and free-water values were calculated within each section. These values were compared between the first-episode and control groups via a $t$ test that was performed for each section separately (Matlab), while adjusting for the age, motion, and TE covariates. False discovery rate (FDR) correction was applied to account for multiple comparisons between the different ROIs. Test results that met the FDR criteria were considered significant. Results that did not meet the FDR criteria yet had a significance level $p<0.05$ were considered trend-level findings. The FDR criteria applies a more strict multiple comparisons correction than the TFCE-based statistical approach that was applied on the white matter skeleton. As a result, the FreeSurfer ROI analysis is likely to have more type II errors (false negatives) than the TBSS analysis, even though the number of the comparisons is lower for the ROI analysis. Nevertheless, the FreeSurfer analysis allows the investigation of gray matter, which is excluded in the TBSS analysis. Furthermore, it avoids between-subject registration issues, which are extremely inaccurate in cortical areas, and thus provides a more robust way to compare subject groups than voxel-based ROI analyses.

The FreeSurfer sections were further combined into four global regions of interest (ROIs): right and left hemisphere white matter, and right and left hemisphere gray matter. The mean free-water value for each ROI was calculated as the average over the sections weighted by each section's volume (estimated by number of voxels). The mean values of each ROI were compared between the first-episode and control groups using a $t$ test (Matlab) while adjusting for the age, motion, and TE covariates. Clustering together all FreeSurfer ROIs that delineated the ventricles formed an additional ROI. The mean MD value for this ventricular ROI was calculated for each subject and compared via a $t$ test.

\section{Results}

\section{Abnormalities in diffusion tensor imaging indices on the white matter skeleton}

Analyzing the diffusion data using a conventional DTI model yielded voxelwise maps of FA and MD for each subject. Comparison of the first-episode group with the control group was restricted to the projection of the maps onto a white matter skeleton to reduce registration errors originating from gross volume changes, such as ventricle sizes (Douaud et al., 2007). The first-episode group had widespread significantly lower FA values than the control group. The FA abnormalities were distributed globally extending through most brain parts (Fig. 1), covering 30.85\% of the left hemisphere white matter and $30.98 \%$ of the right hemisphere white matter. In addition, the first-episode group showed significantly higher MD values in areas that spatially overlapped and extended beyond the FA value changes (Fig. 1), covering $53.61 \%$ of the right hemisphere white matter and $48.78 \%$ of the left hemisphere white matter.

\section{Free-water imaging indices abnormalities on the white matter skeleton}

Analyzing the diffusion data with the free-water methodology yielded an $\mathrm{FA}_{\mathrm{T}}$ map measuring the fractional anisotropy of water in the tissue and a free-water map that measured the fractional volume of extracellular water, i.e., molecules that are not restricted by cellular membranes or other cellular microstructures. The $\mathrm{FA}_{\mathrm{T}}$ and free-water maps were projected onto the same white matter skeleton used for the DTI analysis.

The first-episode group showed significantly lower $\mathrm{FA}_{\mathrm{T}}$ values compared with the control group, in limited brain areas, covering $1.25 \%$ of the left hemisphere white matter and $1.65 \%$ of the right hemisphere white matter (Fig. 2). The affected areas include neuronal connections in the frontal lobe alone, in areas consisting of parts of the superior longitudinal fasciculus bilaterally; the right inferior frontal occipital fasciculus; and the intersection of the 

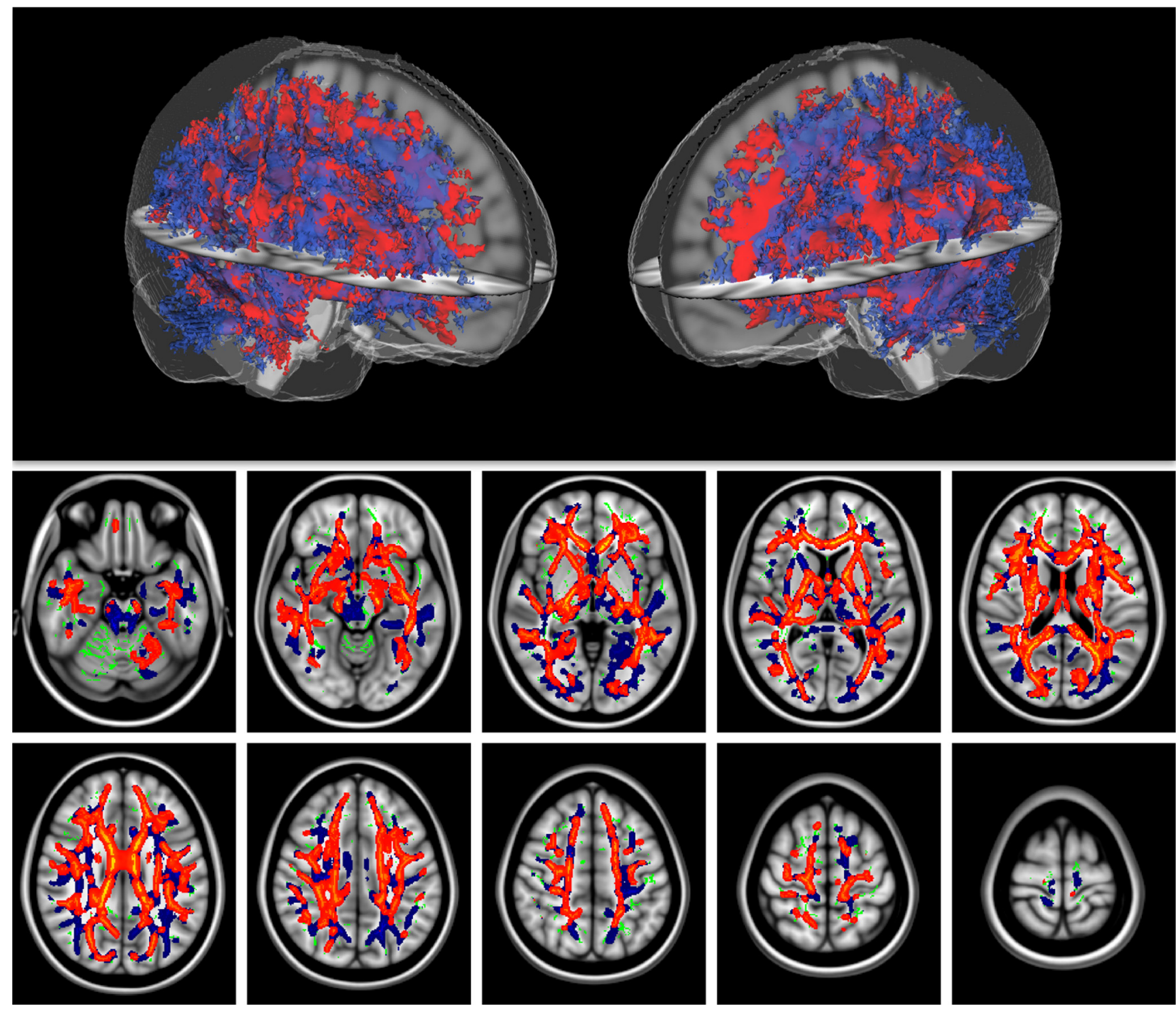

Figure 1. Abnormal DTI values in first-episode patients. Axial slices (bottom) and 3D rendering (top) demonstrate a global pattern for significant FA value decreases (red to yellow) and for significant MD value increases (blue to dark blue) throughout the white matter skeleton (green). The FA map is drawn on top of the MD map and on top of the skeleton.

callosal fibers with the right superior corona radiata. Averaging the $\mathrm{FA}_{\mathrm{T}}$ values within the areas that showed significant group differences, and plotting this mean value against age (Fig. 3) showed that in these brain areas each first-episode subject had lower $\mathrm{FA}_{\mathrm{T}}$ values than control individuals in the same age range, providing a clear separation between the two groups.

Significant increase of free-water values in the first-episode group showed a global pattern spatially overlapping with the MD and FA value changes (compare Figs. 1,2), covering $41.14 \%$ of the left hemisphere white matter and $38.08 \%$ of the right hemisphere white matter. There were no voxels with significant correlation between the free-water imaging measures (free-water and $\mathrm{FA}_{\mathrm{T}}$ maps) and the following variables: the total BPRS rating, the duration of illness, the $\mathrm{CPZ}$ equivalent daily dosage value, and duration of medication.

\section{Whole brain analysis}

As an alternative to the skeletonized analysis, and to extend the comparison of the free-water indices into gray matter areas, we compared the mean free-water values and mean $\mathrm{FA}_{\mathrm{T}}$ in four global ROIs (right and left hemisphere white matter, and right and left hemisphere gray matter) derived from the FreeSurfer sections. The mean free-water values for the first-episode group in all of these ROIs were significantly higher than the mean freewater values of the control group (Fig. 4). The absolute differences in the means were consistent across these ROIs (Table 2), with differences in increase of extracellular fractional volume ranging from 1.1 to $1.6 \%$. Within the control group and within the first-episode group, there were no significant differences in the free-water values when comparing the left and right hemisphere in any of the four ROIs.

We further compared the volume of the ROIs (number of voxels) between the first-episode and control groups, to reveal any atrophy related group differences. There were no significant volume differences in the left gray matter $\left(t_{(33)}=-0.71 ; p=\right.$ $0.48)$, the right gray matter $\left(t_{(33)}=-0.62 ; p=0.54\right)$, the left white matter $\left(t_{(33)}=-0.02 ; p=0.99\right)$, or the right white matter $\left(t_{(33)}=-0.09 ; p=0.93\right)$ ROIs.

Finally we compared the mean MD values averaged over the ventricles, as defined by the FreeSurfer ROIs, to identify potential 

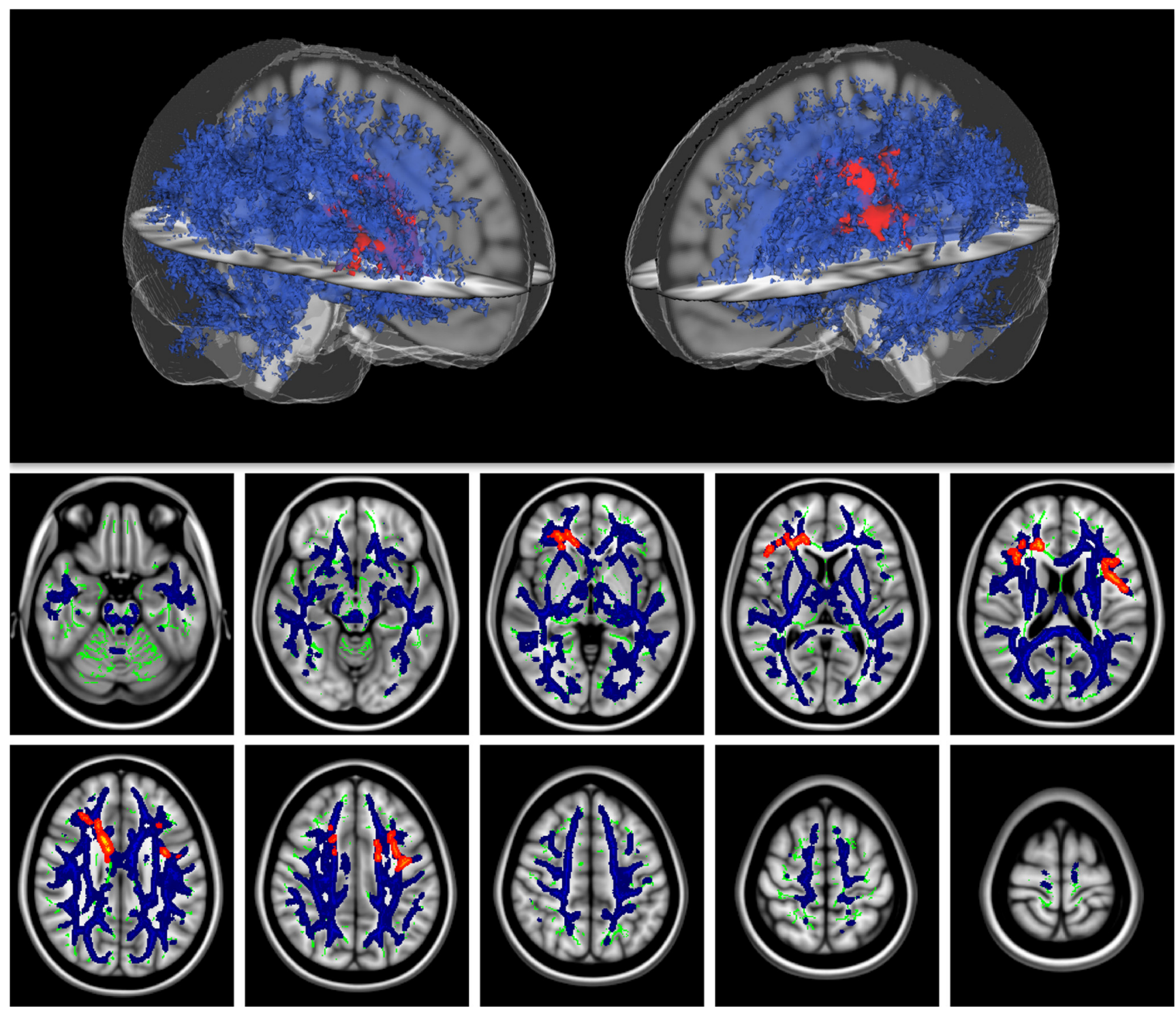

Figure 2. Abnormal free-water values in first-episode patients. Axial slices (bottom) and 3D rendering (top) demonstrate a localized pattern for significant $F A_{T}$ value decreases (red to yellow) in fibers that pass through the frontal lobe. A global pattern for significant free-water value increases (blue to dark blue) is found throughout the white matter skeleton (green). The $F A_{T} m^{m a p ~ i s ~ d r a w n ~}$ on top of the free-water map and on top of the skeleton.

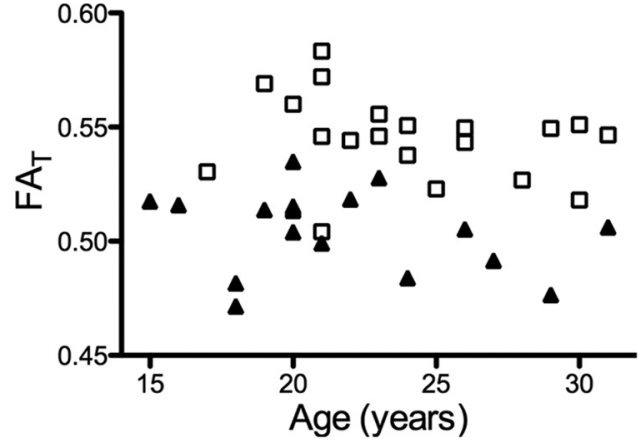

Figure 3. Individual differences in $\mathrm{FA}_{\mathrm{T}}$. Each first-episode patient had lower $F A_{\mathrm{T}}$ values than age-matched controls when averaging within the area that showed significant voxelwise group differences in $\mathrm{FA}_{\mathrm{T}}$. The clear separation between the two groups suggests that the $\mathrm{FA}_{\mathrm{T}}$ measure in this area (marked red to yellow in Fig. 2) is a strong biomarker for schizophrenia. biases such as body temperature differences that may have affected the estimation of diffusivity in general. However, there were no significant differences $\left(t_{(33)}=-0.99 ; p=0.33\right)$ between the groups.

Abnormalities of free-water imaging indices in the gray matter

We looked at each individual gray matter section to see whether the global changes evinced in white matter are also found in the gray matter. The free-water value was increased in the vast majority of the gray matter (64 of 70 gray matter sections) of the first-episode patients compared with the control group. The increase was significant in 6 of 70 sections (minimal $t_{(33)}=3.16$; $p=0.0034$ ), with an additional 26 of 70 sections that had a trend-level group difference (minimal $t_{(33)}=2.07 ; p=0.046$ ). Similar to the white matter analysis, the free-water increase overlapped with a wider extent of MD changes, with 43 of 70 sections that had a significant $\mathrm{MD}$ increase $\left(\right.$ minimal $t_{(33)}=2.30 ; p=$ 0.028 ) and 9 of 70 sections that had a trend-level increase (min- 
imal $\left.t_{(33)}=2.03 ; p=0.05\right)$. There were no atrophy signs of reduced volume in the gray matter sections, with only a single section showing a decrease with a trendlevel significance (left pericalcarine cortex; $\left.t_{(33)}=-2.5951 ; p=0.014\right)$.

Contrary to the free-water volume fraction and MD changes, global changes in $\mathrm{FA}_{\mathrm{T}}$ in the gray matter were not evident. In 39 of 70 gray matter sections of the first-episode subjects, the $\mathrm{FA}_{\mathrm{T}}$ value decreased compared to the control group, of which only a single section had a trendlevel significance level (the left rostral anterior cingulate; $\left.t_{(33)}=-2.54 ; p=0.016\right)$. In the remaining 31 of 70 gray matter sections, the $\mathrm{FA}_{\mathrm{T}}$ value increased, yet only in a single section did this increase have a trend-level significance level (right middle temporal cortex; $t_{(33)}=2.63 ; p=$ $0.013)$. The mean $\mathrm{FA}_{\mathrm{T}}$ values in the left hemisphere gray matter were 0.204 for the controls and 0.200 for the first-episode patients $\left(t_{(33)}=-0.52 ; p=0.65\right)$, and in the right hemisphere were 0.206 for the controls and 0.207 for the first-episode patients $\left(t_{(33)}=0.21 ; p=0.83\right)$. These values are isotropic, yet slightly higher from FA values, due to the extraction of the purely isotropic free-water component.

\section{Discussion}

Consistent with previous reports, we found widespread white matter abnormalities in first-episode patients. Using free-water imaging, we demonstrated for the first time that these abnormalities can be explained by two microstructurally separate components: the extracellular volume and the diffusion anisotropy of water in tissue, $\mathrm{FA}_{\mathrm{T}}$. The key finding here is that the majority of abnormalities in the early stage of schizophrenia are explained by an increase in the extracellular volume, whereas a decrease in $\mathrm{FA}_{\mathrm{T}}$ is limited in its extent to focal areas in the frontal lobe (Fig. 2). Assuming that neuroinflammation and axonal degeneration are the two likely white matter pathologies in schizophrenia, our findings suggest that excessive extracellular volume, reflecting neuroinflammation and not degeneration, is the predominant pathology found at onset.

Neuroinflammation is a dynamic response of the intrathecal immune system to harmful stimuli (Streit, 2006). The response is initiated by the activation of microglia and astrocytes that emit chemicals that induce excessive osmosis of water from the blood into the extracellular space (Schwartz et al., 2006). This mechanism is responsible for the increase in the relative volume of the extracellular space (Sykova and Nicholson, 2008), reflected as an increase of free-water values, whereas the tissue itself, and hence the $\mathrm{FA}_{\mathrm{T}}$ value, remains unchanged. In chronic neuroinflammation, a secondary effect of cellular damage affects the surrounding tissue (Streit, 2006). This is believed to play a key role in the etiology of neurodegenerative disorders, such as multiple sclerosis, where an initial lesion induces inflammation that eventually damages the oligodendrocytes, the myelin sheaths, and, ultimately, the axons and neurons (Weiner and Selkoe, 2002). Such damage in white matter can be reflected by $\mathrm{FA}_{\mathrm{T}}$ changes, which is more specific to tissue changes than the conventional FA measure (Pasternak et al., 2009; Metzler-Baddeley et al., 2012).
Left WM

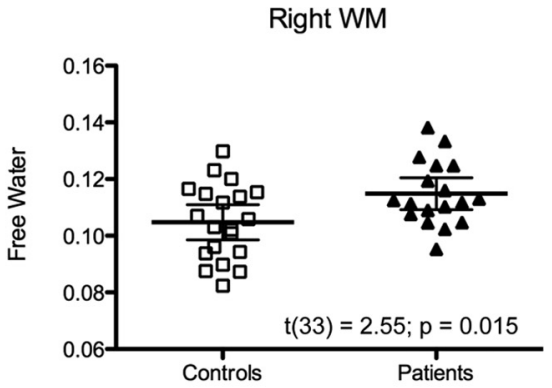

Left GM

Right GM

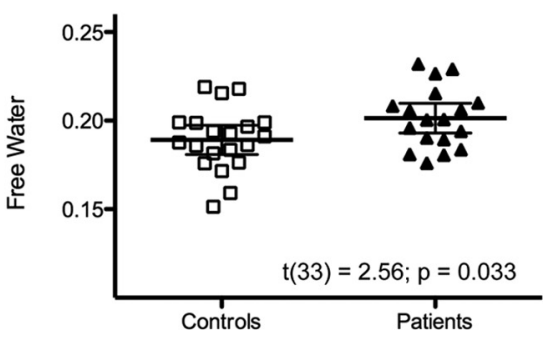

Figure 4. Global differences in free water. Free-water values in patients are globally significantly higher than free-water values in controls. The global pattern of increase is evident both in white and gray matter, in both hemispheres. Black lines represent the mean and the $95 \%$ confidence interval.

Table 2. Global gray and white matter ROIs

\begin{tabular}{lllll}
\hline & \multicolumn{2}{l}{ Mean free water } & & \\
\cline { 2 - 3 } & First episode & Controls & Difference & $t_{(\mathrm{df})} ; p$ \\
\hline Left gray matter & 0.1991 & 0.1826 & 0.0165 & $t_{(33)}=2.48 ; p=0.0184$ \\
Right gray matter & 0.2021 & 0.1885 & 0.0136 & $t_{(33)}=2.23 ; p=0.0331$ \\
Left white matter & 0.1145 & 0.1031 & 0.0115 & $t_{(33)}=2.54 ; p=0.0161$ \\
Right white matter & 0.1154 & 0.1042 & 0.0112 & $t_{(33)}=2.55 ; p=0.0155$ \\
\hline
\end{tabular}

$t_{\text {(df) }}$ is the $t$ statistic and degrees of freedom; $p$ is the significance value.

The extent of excessive extracellular volume we find in the firstepisode patients suggests that schizophrenia pathophysiology may show a similar neurodegenerative pattern in which chronic neuroinflammation eventually causes axonal degeneration and the related deterioration in cognitive abilities. This interpretation, which can be confirmed in longitudinal studies, differs from the current leading theories of schizophrenia etiology, where neuroinflammation is not considered a key component. If, indeed, neuroinflammation precedes degeneration in the disease progression, then our findings suggest that the frontal connections, where the decrease of $\mathrm{FA}_{\mathrm{T}}$ values was found, are the first fibers to show degeneration. This may be related to the fact that these fibers are among those still being myelinated in late adolescence and early adulthood (Tamnes et al., 2010), and thus could be more vulnerable to damage.

The decrease of $\mathrm{FA}_{\mathrm{T}}$ values in frontal white matter areas corresponds well with the literature findings where pathologies in the frontal lobe are considered among the hallmarks of schizophrenia (Cerqueira et al., 2005). In fact, myelin-related abnormalities in schizophrenia in postmortem studies were found mostly within the frontal lobes (Uranova et al., 2007). In addition, a previous metaanalysis of DTI studies of schizophrenia patients reported that areas in the left frontal lobe and left temporal lobe consistently show a decrease of FA values, concluding that there is evidence of localized myelin deficiencies in schizophrenia (Ellison-Wright and Bullmore, 2009). The $\mathrm{FA}_{\mathrm{T}}$ value changes we report here further support this conclusion, as the significant differences between the groups are localized as well. Moreover, when plotting the $\mathrm{FA}_{\mathrm{T}}$ values over age (Fig. 
3), a remarkable separation between the groups appears, which suggests that $\mathrm{FA}_{\mathrm{T}}$ is a strong biomarker for schizophrenia. However, the free-water value differences we report here offer an important addition to the localized theory, asserting that there is a global extracellular process (both in white and gray matter) that occurs at the same time, and possibly precedes local axonal alterations.

A global pattern of white matter abnormalities was noticed in many recent DTI studies of first-episode patients (Kyriakopoulos and Frangou, 2009; Peters et al., 2010), possibly also due to steadily improving imaging and analysis tools (Kubicki and Shenton, 2009). However, it was theorized previously that the pattern reflects a genetic abnormality in the protein pathways controlling myelination, leading to wide-extent myelin alterations (Konrad and Winterer, 2008), not addressing the findings in the chronic stages that usually reveal focal degeneration patterns instead (Ellison-Wright and Bullmore, 2009). Since here we are able to distinguish the contribution of axonal alterations from extracellular changes, we can assert that the global component evinced in the first-episode patients is of an extracellular source, which fits well with the expected effect of neuroinflammation but not with the expected effect of myelin alterations.

Finding histological evidence of neuroinflammation is highly complicated by the fact that this process is dynamic and may not be prevalent in chronic cases (Connor et al., 2011). However, neuroinflammation traces have been reported in subjects who committed suicide during a psychotic event (Steiner et al., 2008). Previous imaging studies demonstrated the involvement of neuroinflammation in schizophrenia as higher binding rates to $\left[{ }^{11} \mathrm{C}\right](R)$-PK11195 (van Berckel et al., 2008), indicating higher rates of activated microglia than in controls. This requires PET scanning, which is expensive, invasive, and has poorer spatial resolution compared to diffusion MRI. Of importance, the diffusion MRI advantages enable longitudinal studies. Neuroinflammation was indirectly suggested by imaging measures such as increased T2 relaxation (Pfefferbaum et al., 1999), magnetization transfer ratio (Peters et al., 2010), and changes in MD (DeLisi et al., 2006; Garver et al., 2008). The sensitivity of DTI measurements to neuroinflammation has been demonstrated in other neurodegenerative diseases, where inflammation is often a key component in the etiology (Weiner and Selkoe, 2002; Cloak et al., 2004; Acosta-Cabronero et al., 2010). In line with these findings, here we report an overlap between free-water value and MD value abnormalities. However, we also show that MD and noncorrected FA values are highly spatially correlated, and thus do not allow separation between neuroinflammation and degeneration that the freewater and $\mathrm{FA}_{\mathrm{T}}$ values provide.

Other pathologies that could explain an increase in the extracellular volume were detected previously in schizophrenia, however never with a global pattern. These include cellular membrane breakdown, abnormally low cell density, and low dendritic number and volume (Selemon et al., 2002, 2003; Segal et al., 2009). There may be a global reduction in perineuronal extracellular matrix; however, so far this has only been reported in medial temporal structures (Pantazopoulos et al., 2010). There may be inflated Virchow-Robin spaces, which can accumulate water pockets in the periventricular space (Wuerfel et al., 2008), yet there are no explicit reports for such findings in schizophrenia. Atrophy is also frequently reported in schizophrenia imaging studies; however, it was not evident in our data. This could be explained by the progressive nature of atrophy (e.g., Whitford et al., 2006), which may not yet be manifested in our first-episode cohort.

Age, gender, and motion differences are additional possible confounds that theoretically could explain systematic increase in the diffusivity for the schizophrenia group. In our data there were no significant differences in age and gender distribution, nor were there significant differences in SNR or motion observed between the two groups (Table 1). Moreover, age and motion parameters were added as control variables in the statistical analysis. Increased T2 relaxation could also bias the estimation; however, overestimation of the free-water volume could be either due to an increased extracellular relative volume, or due to an increase in the T2 relaxation of the extracellular pool. Both options imply changes in the extracellular space that are likely related to inflammation. Therefore, based on currently available evidence, neuroinflammation appears the most likely explanation for the excessive extracellular volume.

The source of neuroinflammation in schizophrenia and in many other neurodegenerative disorders is still under debate. Neuroinflammation could be the result of an infectious disease or an autoimmune response (Streit et al., 2004). It could also be a response of the immune system to a chronic state of mental stress (Muller and Schwarz, 2006) or a response to medication, although here we do not find a correlation between an increase in free-water values and the dosage or duration of antipsychotic medications. The effect of nonpsychotic or nonprescription drugs remains a possible confound. However, many of the psychiatric drugs have an anti-inflammatory effect (Sommer et al., 2012), which might explain why inflammation is less prevalent in chronic cases.

Early detection of inflammation in schizophrenia is therefore an important task in revealing the etiology, especially since neuroinflammation could be reversed with interventions, such as immunosuppressive drugs, which are likely to be more efficient in the early stage of the disease. Our results here suggest that the free-water contrast shows promise as an early stage neuroinflammatory biomarker, which might provide a time window for anti-inflammatory treatment to minimize the possible cascade of neurodegenerative processes, potentially minimizing the associated symptoms. The proposed analysis provides a foundation for future longitudinal studies that further investigate the association between neuroinflammation and degeneration, as demonstrated by the progression of the free-water and the $\mathrm{FA}_{\mathrm{T}}$ parameters. This kind of longitudinal analysis would be important to further understand the etiology of schizophrenia as well as other neurodegenerative disorders.

\section{References}

Acosta-Cabronero J, Williams GB, Pengas G, Nestor PJ (2010) Absolute diffusivities define the landscape of white matter degeneration in Alzheimer's disease. Brain 133:529-539. CrossRef Medline

Aja-Fernandez S, Niethammer M, Kubicki M, Shenton ME, Westin CF (2008) Restoration of DWI data using a Rician LMMSE estimator. IEEE Trans Med Imaging 27:1389-1403. CrossRef Medline

Alexander AL, Lee JE, Lazar M, Field AS (2007) Diffusion tensor imaging of the brain. Neurotherapeutics 4:316-329. CrossRef Medline

Andreasen NC (2010) The lifetime trajectory of schizophrenia and the concept of neurodevelopment. Dialogues Clin Neurosci 12:409-415. Medline

Assaf Y, Pasternak O (2008) Diffusion tensor imaging (DTI)-based white matter mapping in brain research: a review. J Mol Neurosci 34:51-61. CrossRef Medline

Basser PJ, Pierpaoli C (1996) Microstructural and physiological features of tissues elucidated by quantitative-diffusion-tensor MRI. J Magn Reson B 111:209-219. CrossRef Medline

Baumgartner C, Pasternak O, Bouix S, Westin C-F, Rathi Y (2012) Filtered multi-tensor tractography using free water estimation. Paper presented at International Society for Magnetic Resonance in Medicine Meeting, May 2012, Melbourne, Australia.

Cerqueira JJ, Pêgo JM, Taipa R, Bessa JM, Almeida OF, Sousa N (2005) Morphological correlates of corticosteroid-induced changes in prefrontal cortex-dependent behaviors. J Neurosci 25:7792-7800. CrossRef Medline

Cloak CC, Chang L, Ernst T (2004) Increased frontal white matter diffusion is associated with glial metabolites and psychomotor slowing in HIV. J Neuroimmunol 157:147-152. CrossRef Medline 
Connor CM, Crawford BC, Akbarian S (2011) White matter neuron alterations in schizophrenia and related disorders. Int J Dev Neurosci 29:325334. CrossRef Medline

Davis KL, Stewart DG, Friedman JI, Buchsbaum M, Harvey PD, Hof PR, Buxbaum J, Haroutunian V (2003) White matter changes in schizophrenia: evidence for myelin-related dysfunction. Arch Gen Psychiatry 60:443-456. CrossRef Medline

DeLisi LE, Szulc KU, Bertisch H, Majcher M, Brown K, Bappal A, Branch CA, Ardekani BA (2006) Early detection of schizophrenia by diffusion weighted imaging. Psychiatry Res 148:61-66. CrossRef Medline

Doorduin J, de Vries EF, Willemsen AT, de Groot JC, Dierckx RA, Klein HC (2009) Neuroinflammation in schizophrenia-related psychosis: a PET study. J Nucl Med 50:1801-1807. CrossRef Medline

Douaud G, Smith S, Jenkinson M, Behrens T, Johansen-Berg H, Vickers J, James S, Voets N, Watkins K, Matthews PM, James A (2007) Anatomically related grey and white matter abnormalities in adolescent-onset schizophrenia. Brain 130:2375-2386. CrossRef Medline

Ellison-Wright I, Bullmore E (2009) Meta-analysis of diffusion tensor imaging studies in schizophrenia. Schizophr Res 108:3-10. CrossRef Medline

Garver DL, Holcomb JA, Christensen JD (2008) Compromised myelin integrity during psychosis with repair during remission in drug-responding schizophrenia. Int J Neuropsychopharmacol 11:49-61. Medline

Gur Y, Pasternak O, Sochen N (2009) Fast GL (n)-invariant framework for tensors regularization. Int J Comp Vis 85:211-222. CrossRef

Konrad A, Winterer G (2008) Disturbed structural connectivity in schizophrenia primary factor in pathology or epiphenomenon? Schizophr Bull 34:72-92. Medline

Kubicki M, Shenton ME (2009) Diffusion tensor imaging and its application to schizophrenia and related disorders. Diffusion MRI: from quantitative measurement to in vivo neuroanatomy (Johansen-Berg $\mathrm{H}$, Behrens T, eds). London, Academic.

Kubicki M, Park H, Westin CF, Nestor PG, Mulkern RV, Maier SE, Niznikiewicz M, Connor EE, Levitt JJ, Frumin M, Kikinis R, Jolesz FA, McCarley RW, Shenton ME (2005) DTI and MTR abnormalities in schizophrenia: analysis of white matter integrity. Neuroimage 26:1109-1118. CrossRef Medline

Kubicki M, McCarley R, Westin CF, Park HJ, Maier S, Kikinis R, Jolesz FA, Shenton ME (2007) A review of diffusion tensor imaging studies in schizophrenia. J Psychiatr Res 41:15-30. CrossRef Medline

Kyriakopoulos M, Frangou S (2009) Recent diffusion tensor imaging findings in early stages of schizophrenia. Curr Opin Psychiatry 22:168-176. CrossRef Medline

Laan W, Smeets H, de Wit NJ, Kahn RS, Grobbee DE, Burger H (2009) Glucocorticosteroids associated with a decreased risk of psychosis. J Clin Psychopharmacol 29:288-290. CrossRef Medline

Lieberman JA (1999) Is schizophrenia a neurodegenerative disorder? A clinical and neurobiological perspective. Biol Psychiatry 46:729-739. CrossRef Medline

Ling J, Merideth F, Caprihan A, Pena A, Teshiba T, Mayer AR (2012) Head injury or head motion? Assessment and quantification of motion artifacts in diffusion tensor imaging studies. Hum Brain Mapp 33:50-62. CrossRef Medline

Metzler-Baddeley C, Jones DK, Belaroussi B, Aggleton JP, O’Sullivan MJ (2011) Frontotemporal connections in episodic memory and aging: a diffusion MRI tractography study. J Neurosci 31:13236-13245. CrossRef Medline

Metzler-Baddeley C, O’Sullivan MJ, Bells S, Pasternak O, Jones DK (2012) How and how not to correct for CSF-contamination in diffusion MRI. Neuroimage 59:1394-1403. CrossRef Medline

Muller N, Schwarz M (2006) Schizophrenia as an inflammationmediated dysbalance of glutamatergic neurotransmission. Neurotox Res 10:131-148. CrossRef Medline

Nichols TE, Holmes AP (2002) Nonparametric permutation tests for functional neuroimaging: a primer with examples. Hum Brain Mapp 15:1-25. CrossRef Medline

Pantazopoulos H, Woo TU, Lim MP, Lange N, Berretta S (2010) Extracellular matrix-glial abnormalities in the amygdala and entorhinal cortex of subjects diagnosed with schizophrenia. Arch Gen Psychiatry 67:155-166. CrossRef Medline

Pasternak O, Sochen N, Gur Y, Intrator N, Assaf Y (2009) Free water elim- ination and mapping from diffusion MRI. Magn Reson Med 62:717-730. CrossRef Medline

Pasternak O, Sochen N, Basser PJ (2010) The effect of metric selection on the analysis of diffusion tensor MRI data. Neuroimage 49:2190-2204. CrossRef Medline

Pasternak O, Shenton ME, Westin CF (2012) Estimation of extracellular volume from regularized multi-shell diffusion MRI. Med Image Comput Comput Assist Interv LNCS 7511:305-312.

Peters BD, Blaas J, Haan LD (2010) Diffusion tensor imaging in the early phase of schizophrenia: what have we learned? J Psychiatr Res 44:993-1004. Medline

Pfefferbaum A, Sullivan EV, Hedehus M, Moseley M, Lim KO (1999) Brain gray and white matter transverse relaxation time in schizophrenia. Psychiatry Res 91:93-100. CrossRef Medline

Pierpaoli C, Jones DK (2004) Removing CSF contamination in brain DTMRIs by using a two-compartment tensor model. Paper presented at International Society for Magnetic Resonance in Medicine Meeting, May 2004, Kyoto, Japan.

Potvin S, Stip E, Sepehry AA, Gendron A, Bah R, Kouassi E (2008) Inflammatory cytokine alterations in schizophrenia: a systematic quantitative review. Biol Psychiatry 63:801-808. CrossRef Medline

Schwartz M, Butovsky O, Brück W, Hanisch UK (2006) Microglial phenotype: is the commitment reversible? Trends Neurosci 29:68-74. CrossRef Medline

Segal D, Schmitz C, Hof PR (2009) Spatial distribution and density of oligodendrocytes in the cingulum bundle are unaltered in schizophrenia. Acta Neuropathol 117:385-394. CrossRef Medline

Selemon LD, Kleinman JE, Herman MM, Goldman-Rakic PS (2002) Smaller Frontal Gray Matter Volume in Postmortem Schizophrenic Brains. Am J Psychiatry 159:1983-1991. CrossRef Medline

Selemon LD, Mrzljak J, Kleinman JE, Herman MM, Goldman-Rakic PS (2003) Regional specificity in the neuropathologic substrates of schizophrenia: a morphometric analysis of Broca's area 44 and area 9. Arch Gen Psychiatry 60:69-77. CrossRef Medline

Smith SM, Nichols TE (2009) Threshold-free cluster enhancement: addressing problems of smoothing, threshold dependence and localisation in cluster inference. Neuroimage 44:83-98. CrossRef Medline

Smith SM, Jenkinson M, Johansen-Berg H, Rueckert D, Nichols TE, Mackay CE, Watkins KE, Ciccarelli O, Cader MZ, Matthews PM, Behrens TE (2006) Tract-based spatial statistics: voxelwise analysis of multi-subject diffusion data. Neuroimage 31:1487-1505. CrossRef Medline

Sommer IE, de Witte L, Begemann M, Kahn RS (2012) Nonsteroidal antiinflammatory drugs in schizophrenia: ready for practice or a good start? A meta-analysis. J Clin Psychiatry 73:414-419. CrossRef Medline

Song SK, Sun SW, Ju WK, Lin SJ, Cross AH, Neufeld AH (2003) Diffusion tensor imaging detects and differentiates axon and myelin degeneration in mouse optic nerve after retinal ischemia. Neuroimage 20:1714-1722. CrossRef Medline

Steiner J, Bielau H, Brisch R, Danos P, Ullrich O, Mawrin C, Bernstein HG, Bogerts B (2008) Immunological aspects in the neurobiology of suicide: elevated microglial density in schizophrenia and depression is associated with suicide. J Psychiatr Res 42:151-157. CrossRef Medline

Streit WJ (2006) Microglial senescence: does the brain's immune system have an expiration date? Trends Neurosci 29:506-510. CrossRef Medline

Streit WJ, Mrak RE, Griffin WS (2004) Microglia and neuroinflammation: a pathological perspective. J Neuroinflammation 1:14. CrossRef Medline

Syková E, Nicholson C (2008) Diffusion in brain extracellular space. Physiol Rev 88:1277-1340. CrossRef Medline

Tamnes CK, Ostby Y, Fjell AM, Westlye LT, Due-Tonnessen P, Walhovd KB (2010) Brain maturation in adolescence and young adulthood: regional age-related changes in cortical thickness and white matter volume and microstructure. Cereb Cortex 20:534-548. CrossRef Medline

Uranova NA, Vostrikov VM, Vikhreva OV, Zimina IS, Kolomeets NS, Orlovskaya DD (2007) The role of oligodendrocyte pathology in schizophrenia. Int J Neuropsychopharmacol 10:537-545. CrossRef Medline

van Berckel BN, Bossong MG, Boellaard R, Kloet R, Schuitemaker A, Caspers E, Luurtsema G, Windhorst AD, Cahn W, Lammertsma AA, Kahn RS (2008) Microglia activation in recent-onset schizophrenia: a quantitative (R)-[11C]PK11195 positron emission tomography study. Biol Psychiatry 64:820-822. CrossRef Medline

Ventura J, Nuechterlein KH, Subotnik KL, Gutkind D, Gilbert EA (2000) Symptom dimensions in recent-onset schizophrenia and mania: a prin- 
cipal components analysis of the 24-item Brief Psychiatric Rating Scale. Psychiatry Res 97:129-135. CrossRef Medline

Wang Y, Wang Q, Haldar JP, Yeh FC, Xie M, Sun P, Tu TW, Trinkaus K, Klein RS, Cross AH, Song SK (2011) Quantification of increased cellularity during inflammatory demyelination. Brain 134:3590-3601. CrossRef Medline

Weiner HL, Selkoe DJ (2002) Inflammation and therapeutic vaccination in CNS diseases. Nature 420:879-884. CrossRef Medline
Whitford TJ, Grieve SM, Farrow TF, Gomes L, Brennan J, Harris AW, Gordon E, Williams LM (2006) Progressive grey matter atrophy over the first 2-3 years of illness in first-episode schizophrenia: a tensorbased morphometry study. Neuroimage 32:511-519. CrossRef Medline

Wuerfel J, Haertle M, Waiczies H, Tysiak E, Bechmann I, Wernecke KD, Zipp F, Paul F (2008) Perivascular spaces-MRI marker of inflammatory activity in the brain? Brain 131:2332-2340. CrossRef Medline 